\title{
Role of neo-adjuvant hormonal therapy in the treatment of breast cancer: a review of clinical trials
}

This article was published in the following Dove Press journal:

International Journal of General Medicine

3 July 2009

Number of times this article has been viewed

\author{
Catherine Abrial ${ }^{1,2}$ \\ Xavier Durando ${ }^{1,2}$ \\ Marie-Ange Mouret-Reynier ${ }^{1,2}$ \\ Emilie Thivat ${ }^{1,2}$ \\ Mathilde Bayet-Robert ${ }^{1,2}$ \\ Béatrice Nayl' \\ Pascale Dubray' \\ Christophe Pomel' \\ Philippe Chollet ${ }^{1,2,3}$ \\ F Penault-Llorca ${ }^{1,2}$ \\ 'Centre Jean Perrin, ${ }^{2}$ EA 4231 , \\ Université d'Auvergne, ${ }^{3}$ Centre \\ d'Investigation Clinique, \\ Clermont-Ferrand, France
}

\begin{abstract}
The clinical benefits of endocrine therapy for patients with hormonosensitive breast cancer are well established. For many years, 5 years of tamoxifen was the gold standard of adjuvant treatment. The recent development of new endocrine agents provides physicians with a more effective therapeutic approach. Nevertheless, the success of neoadjuvant endocrine therapy is much more recent and less reported in the literature. This article reviews the studies published about neoadjuvant endocrine treatment (tamoxifen and aromatase inhibitors). According to the literature, neoadjuvant endocrine therapy seems to be effective. In contrast to neoadjuvant chemotherapy, neoadjuvant endocrine therapy is well tolerated, with very few patients having to discontinue the treatment because of side effects. It does not constitute a standard treatment but could have potential for elderly women with operable, hormonosensitive, well differentiated and slowly progressing (SBR I) tumor or for patients with lobular MSBR 1 carcinoma (low chemosensitivity). The newer generation of aromatase inhibitors (letrozole, anastrozole, exemestane) appears to be more active (in terms of overall response rates and conservative surgery rate) than tamoxifen. Patients with an estrogen receptor Allred score of 6 and over are more likely to respond and gain a clinical benefit. The optimal duration of neoadjuvant therapy has not yet been investigated in detail. These preliminary results should be confirmed by further studies.

Keywords: aromatase inhibitors, breast cancer, endocrine therapy, neoadjuvant, tamoxifen
\end{abstract}

\section{Background History of endocrine therapy}

The use of endocrine therapy for breast cancer dates back to the nineteenth century, when oopherectomy was first shown to cause regression of advanced breast cancer in premenopausal women. ${ }^{1}$ During the twentieth century, endocrine therapies evolved to include adrenalectomy, hypophysectomy, estrogens and androgens. In the early 1970s, tamoxifen, a competitive antagonist-agonist, was introduced, and the management of hormone-receptor breast cancer was changed. For many years, 5 years of tamoxifen was the gold standard of adjuvant treatment and has largely contributed to the effectiveness of such therapy. The recent development of new endocrine agents - the newer generation aromatase inhibitors: letrozole, anastrozole and exemestane - provides physicians with a more effective therapeutic approach, in metastatic disease or in the adjuvant setting of breast cancer patients. ${ }^{2}$

\section{Mechanism of action of tamoxifen}

Tamoxifen, a selective estrogen receptor (ER) modulator, is a competitive inhibitor of estradiol binding to the ER. It has mixed agonist and antagonist effects. The agonist 
effects of tamoxifen can be beneficial, since they may help prevent bone demineralization, but are also detrimental in that they are associated with increased risks of uterine cancer and thromboembolism, and may play a role in development of tamoxifen resistance. ${ }^{3-6}$

\section{Mechanism of action of aromatase inhibitors}

In the premenopausal woman, estrogen synthesis takes place mainly in the ovaries. After menopause, estrogens are synthetized mainly by conversion of androgenic substrates via the aromatase enzyme in the skin, fat, and muscle. The aromatase enzyme has been demonstrated to be present in peripheral tissues as well as in breast tissue, and in breast tumor tissue. The aromatase inhibitors suppress plasma estrogen levels by inhibiting or inactivating aromatase, and therefore have none of the agonist activities of tamoxifen. ${ }^{7}$

\section{Classification of aromatase inhibitors}

Aromatase inhibitors are divided into two categories: type 1 inhibitors, which are steroidal analogues of androstenedione and bind irreversibly to its site of action; and type 2 inhibitors, which are nonsteroidal, and bind reversibly to a heme group in the activation site of the enzyme. Aromatase inhibitors are further characterized as first-, second- and third-generation according to the chronological order of their clinical development (Table 1).

The first-generation aromatase inhibitor, aminogluthetimide (Orimetene ${ }^{\circledR}$ ), became available for use in treatment of breast cancer in the late 1970s. The second-generation aromatase inhibitors include formestane $\left(\right.$ Lentaron $\left.^{\circledR}\right)$, a type 1 inhibitor, fadrozole and roglethimide, type 2 inhibitors. While each of these has exhibited clinical efficacy, significant side effects limited their utility. ${ }^{8-10}$ The third-generation inhibitors were developed in the early 1990s, and include the type 1 inhibitor exemestane $\left(\operatorname{Aromasin}^{\circledR}\right)$, as well as the type II inhibitors anastrozole (Arimidex ${ }^{\circledR}$ ) and letrozole $\left(\right.$ Femara $\left.^{\circledR}\right)$. These three agents have demonstrated high promise for their effects in the metastatic, adjuvant and neoadjuvant settings. ${ }^{\text {? }}$

Until recently, neoadjuvant therapy of breast cancer has used predominantly cytotoxic chemotherapy. Neoadjuvant therapy reduces the size of bulky tumors and a conservative surgery can be proposed to the patients instead of a mastectomy. Endocrine treatment is now emerging as an attractive alternative in hormone receptor positive postmenopausal and mainly elderly women, many of whom could not tolerate the toxicity of chemotherapy. ${ }^{11}$ So, the success of neoadjuvant endocrine therapy is much more recent and less reported in the literature. Respective indications of chemotherapy and hormone therapy as primary treatment are not fully established.

Thus, our aim was to review the clinical data of the main published studies using neoadjuvant hormonotherapy and to compare tamoxifen to letrozole, anastrozole and exemestane.

\section{Studies with tamoxifen (see Table 2)}

Neoadjuvant endocrine therapy was initially assessed in elderly patients with locally advanced breast carcinoma. ${ }^{12}$ In early studies, tamoxifen was used, but patients were not selected on the basis of patient selection with positive ER or progesterone receptor (PgR) breast cancers to identify those most likely to respond.

Many phase II studies were conducted, ${ }^{13}$ but only a few randomized phase III studies have been published. ${ }^{14-18}$ The comparison was mainly tamoxifen as sole therapy versus successive classical treatments. This approach does not correspond to the classical definitions of a neoadjuvant approach and furthermore is not accepted ethically in all countries.

Tan et al conducted a prospective randomized phase II study in 108 locally advanced primary breast cancer and premenopausal patients. ${ }^{14}$ This trial compared multimodal therapy (neoadjuvant chemotherapy, modified radical mastectomy, postoperative radiotherapy and adjuvant tamoxifen) versus tamoxifen alone. After a median follow-up of 52 months, there was no statistically significant difference in the rates of overall and disease-free survival between the two therapy groups. However, the time to first locoregional failure was significantly shorter in the hormone therapy alone group than in the multimodal therapy group.

Some other randomized trials have compared primary endocrine therapy with tamoxifen alone with surgery \pm tamoxifen

Table I Classification of aromatase inhibitors

\begin{tabular}{llll}
\hline & First generation & Second generation & Third generation \\
\hline Nonsteroidal & Aminoglutethimide & Rogletimide, fadrozole, & Anastrozole, letrozole, vorozole, \\
Steroidal & & formestane & exemestane \\
\hline
\end{tabular}




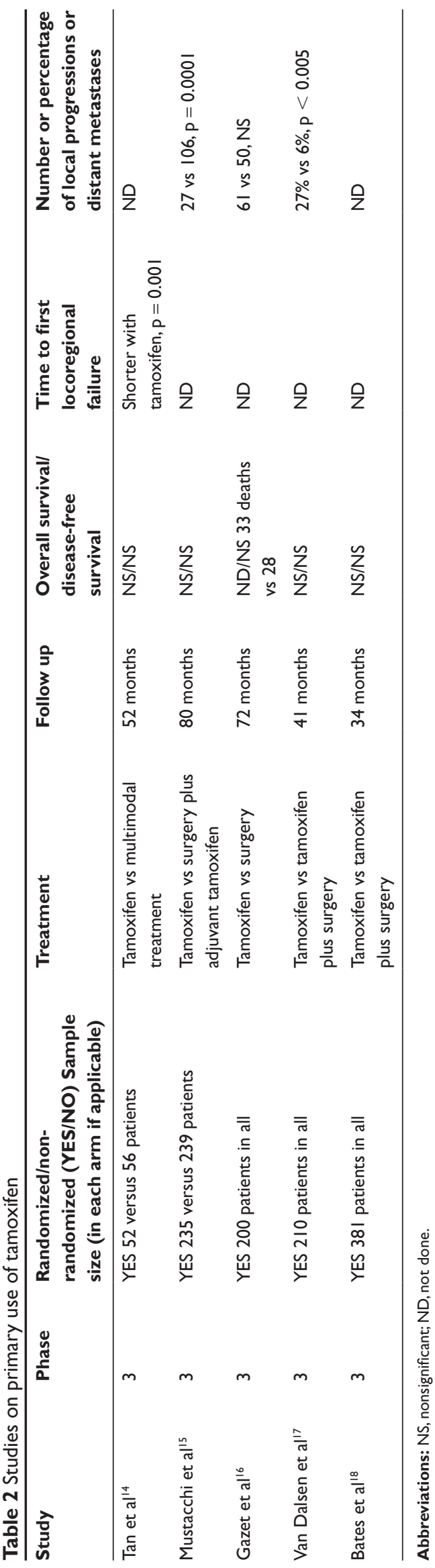

in elderly patients. ${ }^{15-18}$ In two of the studies, ${ }^{15,16}$ tamoxifen was compared with immediate surgery alone, and in the other two, tamoxifen was compared with surgery and tamoxifen. ${ }^{17,18}$ After median follow-up of 34 to 72 months, there were no statistically significant differences in the rates of overall and/or disease-free survival between the two therapy groups. However, again, the time to first locoregional reccurrence was significantly shorter in the tamoxifen alone arm, as would be expected.

This approach of tamoxifen alone is predominant in the United Kingdom and in some Latin European countries, often linked to social and financial considerations. However, it should be linked to positive hormone receptors, as evidently patients with negative receptors do not benefit from this therapy. Furthermore a local relapse is not an incidental event, and can be avoided in most patients.

Another strategy is the true neoadjuvant approach to initiate endocrine therapy in order to prepare a secondary local treatment, with the objective of breast conservation, as has been shown with neoadjuvant chemotherapy. It is recognized that steroid receptor positivity is predictive of response to hormonal manipulation, as well as in adjuvant treatment ${ }^{19}$ or in a palliative setting. ${ }^{20}$ So it seemed possible to propose a neoadjuvant hormonal treatment for women with a tumor that was too large to be treated by conservative surgery (generally $3 \mathrm{~cm}$ in diameter, or more) and who met the criterion of response to hormonal treatment. Mauriac et $\mathrm{al}^{21}$ have published a retrospective study of 199 women aged from 50 to 70 years treated by neoadjuvant tamoxifen and whose tumor expressed steroid receptor positivity. Conserving surgery rates after neoadjuvant tamoxifen were $54 \%$ and $44 \%$ for operable and for locally advanced tumors, respectively. This approach, which is currently used in elderly patients, seems to be applicable in younger women. ${ }^{21}$ These younger women should have been first menopaused by surgery, radiotherapy or with LH-RH analogues.

In Edinburgh, small studies have been performed comparing neoadjuvant tamoxifen with aromatase inhibitors. Although patients were not randomized and the numbers were small, impressive results were achieved. As reported by Dixon et al, ${ }^{11} 46 \%$ of patients treated with tamoxifen had reductions in tumor volume of more than $50 \%$ as assessed by ultrasound scan, versus $88 \%$ of patients treated with letrozole and $78 \%$ of patients treated with anastrozole.

A potential problem when using tamoxifen as neoadjuvant therapy is the long time period required to reach steady state plasma levels, up to 5 weeks. In contrast, the newer aromatase 
inhibitors build up rapidly, reaching therapeutic concentrations within days. ${ }^{11}$

\section{Studies with letrozole (see Table 3)}

The first reported study to investigate the neoadjuvant use of letrozole in patients with untreated locally advanced or large operable breast cancer was conducted by Dixon et $\mathrm{al}^{22}$ The demonstration that letrozole was superior to megestrol acetate $\left(\right.$ Megace $\left.^{\circledR}\right)$ and aminoglutethimide in second-line treatment supports the belief that the neoadjuvant situation is amongst the best to investigate efficacy of letrozole. In a phase I study, Dixon et al treated 24 postmenopausal patients with either 2.5 or $10 \mathrm{mg}$ of letrozole during 3 months to investigate its efficacy used as primary neoadjuvant therapy. All 24 patients were ER-positive. There were 5 complete clinical responses (CR) and 7 partial clinical responses (PR) in the patients treated with $2.5 \mathrm{mg}$ letrozole, and 9 partial responses and 3 patients with stable disease in patients treated with $10 \mathrm{mg}$ letrozole. There was no significant difference in clinical response between the two doses. Assessed by ultrasound and mammography, the 12 patients treated with $2.5 \mathrm{mg}$ had 1 complete response, 9 partial responses and 2 had no change. In the 12 patients treated with $10 \mathrm{mg}$ letrozole, there were 8 partial responses and 4 with no change. One patient treated with the $2.5 \mathrm{mg}$ dose had a complete clinical and pathological response. This study demonstrates high response rates in patients treated with letrozole at either $2.5 \mathrm{mg}$ or $10 \mathrm{mg}$ dose, with significant numbers of patients achieving complete clinical responses within 3 months. Besides, this short period of treatment reduced tumor volume such that 15 patients who, at treatment onset, were considered to require mastectomy, were eligible for breast conservation after 3 months. All patients then underwent successful conservation surgery and only one adverse event was reported throughout the whole 3-month course of treatment (pale stools). This short term study cannot determine whether neoadjuvant letrozole treatment is associated with any survival or disease-free survival benefit. These results on responses should be confirmed in further studies, because several weeks of letrozole are needed before a therapeutic steady-state is reached, because of the pharmacokinetics of this drug.

Miller et al ${ }^{23}$ conducted a study in postmenopausal women with large primary ER-rich ( $>20 \mathrm{fmol} / \mathrm{mg}$ protein) breast cancers. They had been treated neoadjuvantly with either letrozole ( $2.5 \mathrm{mg}$ daily, $\mathrm{n}=12$ or $10 \mathrm{mg}$ daily, $\mathrm{n}=12$ ), anastrozole ( $1 \mathrm{mg}$ daily, $\mathrm{n}=12$ or $10 \mathrm{mg}$ daily, $\mathrm{n}=11$ ), or tamoxifen (40 mg daily, $n=24)$. After 3 months of treatment, the two aromatase inhibitors were more likely to produce

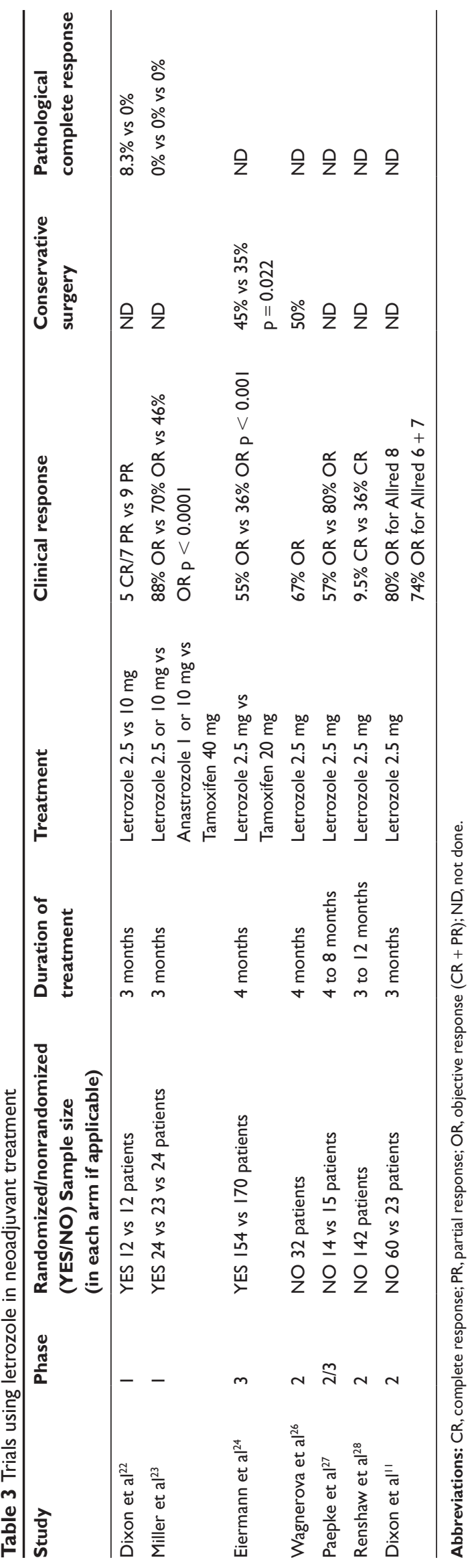


major tumor shrinkages ( $>50 \%$ reduction in volume): 88 and $70 \%$ for letrozole and anastrozole, respectively, compared with tamoxifen (46\%). This difference between aromatase inhibitors and tamoxifen was significant $(\mathrm{p}<0.0001)$. For pathological responses, most tumors treated with aromatase inhibitors demonstrated a partial pathological response; the only tumor showing a complete pathological response was after treatment with letrozole. No adverse events were reported. Tumors from the same cohorts were also stained with antibodies against Ki67 (a marker of cell cycle/proliferation) and PgR (a marker of estrogen action). Immunohistochemical staining for $\mathrm{Ki} 67$ and $\mathrm{PgR}$ provided objective measures of major biological effects of therapy and differences between the aromatase inhibitors and tamoxifen. Letrozole and anastrozole treatment was associated with a reduction in Ki67 staining. This could be interesting since a reduction in Ki67 staining means a reduction of tumor proliferation. This effect was present irrespective of whether there was a clinical/pathological response. Similar reductions in Ki67 score were reported after treatment with tamoxifen but occasionally paradoxal increases in Ki67 score were seen. Although the rise was not associated with clinical/pathological response, this effect could be due to the accumulation of tamoxifen-resistant highly proliferative cells. Treatment with letrozole and anastrozole was associated to fall in score of $\mathrm{PgR}$ in positive patients. In contrast, treatment with tamoxifen was associated with a decrease in PgR in only 4 of 20 evaluable cases; the most common effect was an increase in PgR staining. Since PgR is regarded as a marker of a functional estrogen signaling pathway, the effect was consistent with the estrogen deprivation mechanism of action of these aromatase inhibitors.

These good results have led to further studies. A randomized, double-blind, multicenter study (P024 trial) was conducted to compare the anti-tumor activity of letrozole versus tamoxifene in 337 postmenopausal women with ER and/or PgR positive primary untreated breast cancer. ${ }^{24}$ Patients were randomly assigned once daily treatment with either letrozole $2.5 \mathrm{mg}$ or tamoxifene $20 \mathrm{mg}$ for 4 months. At baseline, none of these patients was considered candidate for breast-conserving surgery (BCS) and $14 \%$ of the patients were considered inoperable. The primary endpoint was to compare overall objective response $(\mathrm{CR}+\mathrm{PR})$ determined by clinical palpation. Secondary endpoints included overall objective response on ultrasound and mammography and the number of patients who qualified for BCS. The overall objective response rate was statistically significantly superior in the letrozole group, $55 \%$ compared to tamoxifen, $36 \%$
( $\mathrm{p}<0.001)$. Secondary endpoints of ultrasound response, $35 \%$ versus $25 \%(\mathrm{p}=0.042)$, mammographic response, $34 \%$ versus $16 \%$ ( $\mathrm{p}<0.001)$, and $\mathrm{BCS}, 45 \%$ versus $35 \%$ ( $p=0.022$ ) between the letrozole and tamoxifen groups, respectively, showed letrozole to be significantly superior. Moreover, both treatments were well tolerated.

Tumor response in this study was related to ER and PgR status. ${ }^{25}$ There were significantly more responses in patients subsequently confirmed to have ER-positive tumors than in patients who on subsequent testing had ER-negative tumors. In each of the ER categories, response rates were higher for letrozole than tamoxifen. Besides, Ellis et al have studied the expression of erbB1 and erbB2 according to the tumors. There was a high difference in response rates in tumors that were ER-positive and also overexpressed erbB1 and/or erbB2, with an $88 \%$ response rate in this group for letrozole versus a $21 \%$ response rate to tamoxifen $(p=0.0004)$. In contrast, in ER-positive tumors which did not overexpress erbB1 and/or erbB2, response rates were similar: 54\% for letrozole versus $42 \%$ for tamoxifen ( $p=0.078$ ).

Another study has been reported by Wagnerova et $\mathrm{al}^{26}$ Twenty-two postmenopausal ER- and/or PgR-positive women in clinical stage IIIB were treated with neoadjuvant oral letrozol $2.5 \mathrm{mg}$ for 4 months. The primary endpoint was to evaluate overall objective response $(\mathrm{CR}+\mathrm{PR})$ determined by clinical examination. Wagnerova et al reported an overall response rate of $67 \%$, with $8 \% \mathrm{CR}$. These results are similar to those of Eiermann et al. ${ }^{24}$

As the overall objective response rate of the P024 study was high with 4 months of letrozole, Paepke et $\mathrm{al}^{27}$ wanted to test the hypothesis of whether longer treatment would further increase the benefit for the patients. Thus, an open clinical trial was conducted with a total of 33 patients. They received letrozole $2.5 \mathrm{mg}$ daily for a minimum of 4 and a maximum of 8 months prior to surgery. Paepke et al reported that a longer treatment resulted in a statistically significant increase in tumor size reduction $(\mathrm{p}=0.0393)$. Moreover, $90 \%$ of the patients receiving pre-operative treatment longer than 4 months had $\mathrm{CR}$ or PR compared to $57 \%$ of patients with $\mathrm{CR}$ or $\mathrm{PR}$ receiving treatment up to 4 months, suggesting that prolongation of the neoadjuvant endocrine treatment might present an encouraging therapeutic option. In the same way, Renshaw et $\mathrm{al}^{28}$ conducted a study in which 142 postmenopausal women were enrolled to receive letrozole $2,5 \mathrm{mg}$ per day. Clinical response was assessed at 3 months. Nonresponders and patients whose tumors had become operable with a conservative surgery proceeded to surgery. The others continued letrozole for 
at least a further 3 months. Some of these were still taking letrozole at 12 months. At 3 months, there were $9.5 \%$ CR, by 6 months, $29 \%$ and by 12 months $36 \%$. The authors concluded that patients whose tumors are responding to letrozole at 3 months can expect further reduction in tumor volume with continued treatment.

In a newer Edinburgh study, ${ }^{11} 83$ patients were treated for 3 months with neoadjuvant letrozole. They were subdivided according to their ER Allred score. ${ }^{29}$ This score varies from 0 to 8 , according to the intensity of positive tumor cells and to the proportion of positive-staining tumor cells. Dixon et al correlated clinical and ultrasound responses and change in tumor volumes in these patients in relation to the ER Allred score. Sixty of the tumors were ER category 8 and 23 were category 6 or 7. Response rates were similar in ER categories 8 and $6+7$ ( $80 \%$ and $74 \%$, respectively), but there was a greater percentage reduction in tumor volume in patients whose tumors had the highest ER level. This difference was significant.

\section{Studies with anastrozole (see Table 4)}

Dixon et $\mathrm{al}^{30}$ evaluated, in a phase I study, the toxicity of anastrozole as neoadjuvant therapy in postmenopausal women with ER-rich (Allred score from 5 to 8), locally advanced or large $(>3 \mathrm{~cm})$, operable breast cancers. Data on efficacy of the treatment were also reported. Twenty-four eligible patients were recruited into the study and received either $1 \mathrm{mg}$ or $10 \mathrm{mg}$ of anastrozole daily over a 3-month period. There were no serious adverse events, and only 1 patient withdrew because of headaches, depression, and tiredness. Median reductions in tumor volumes as measured by ultrasound for those patients with a measurable 12-week assessment were $80.5 \%$ and $69.6 \%$ for anastrozole ( 1 and $10 \mathrm{mg}$, respectively) after 12 weeks of treatment and $75.5 \%$ when both doses were grouped together. Besides, of these patients, 11 of 12 given $1 \mathrm{mg}$ and 7 of 11 (1 patient eligible for the study withdrew because of adverse events) given $10 \mathrm{mg}$ of anastrozole were found on ultrasound to have a $>50 \%$ reduction in tumor volume after 12 weeks of treatment. Of the 17 patients who would have required a mastectomy at initiation of treatment, 15 were suitable for conservative surgery after anastrozole treatment. In general, treatment was well tolerated in this study.

The tumors treated in this study have recently been stained for erbB2 or Her2. ${ }^{31}$ Her2 is an oncogene which belongs to the growth factor receptors family with tyrosin kinase activity. The expression of the protein appears in $15 \%$ to $30 \%$ of breast cancer. In 22 of 24 patients 
treated in this study, erbB2 status prior to treatment, and Ki67 and PgR before and after treatment were assessed. There were 6 erbB2 $3+$ tumors, the other 16 tumors being either negative or $1+$. There was no difference in clinical response in relation to erbB2 status and changes in $\mathrm{Ki} 67$ and PgR did not differ between the two groups. These are the first data demonstrating the clinical and the biological effectiveness of anastrozole in erbB2-positive, ER-positive breast cancers.

Milla-Santos et al $^{32}$ treated 112 hormone-dependent locally advanced breast cancer postmenopausal women with anastrozole (1 mg) during 3 months. They reported 55\% CR and $29 \%$ PR. Patients with a PR or a CR $(n=93)$ underwent surgery. Among these patients, Milla-Santos et al reported $12 \%$ of pathological complete response (disappearance of all tumoral spread) and $71 \%$ of pathological partial response ( $51 \%$ to $99.9 \%$ reduction in tumoral spread). Patients who showed no response to neoadjuvant treatment underwent radiotherapy. No adverse events were reported.

We should mention for the record the study of Miller et $\mathrm{al}^{23}$ (see previous discussion on letrozole).

Smith and Dowsett ${ }^{33}$ reported a multicenter, randomized, double-blind clinical trial: the Immediate Preoperative Arimidex Alone or in Combination with Tamoxifen (IMPACT) study. It compared anastrozole $1 \mathrm{mg}$ daily versus tamoxifen $20 \mathrm{mg}$ daily versus anastrozole plus tamoxifen. Three hundred and thirty postmenopausal patients with large or operable ER- and/or PgR-positive breast cancers, or potentially operable but locally advanced, were recruited. In this study, treatment was for 3 months and patients continued on the same endocrine treatment as adjuvant therapy for 5 years if they responded. Primary endpoints were objective tumor response rates, with secondary endpoints being breastconserving rate and assessment of key biological markers including proliferation, hormone receptors and apoptotic rate. ${ }^{11}$ Smith and Dowsett reported a similar clinical response rate in the anastrozole arm (37\%) in comparison with the tamoxifen arm (36\%) or in the combination arm $(39 \%)$. Patients who had received anastrozole underwent a significantly more conservative surgery than these who had received tamoxifen (46 versus 22\%, p=0.03). In erbB2-positive tumor patients, there was a tendency for the clinical response rate to be increased in anastrozole arm compared to tamoxifen arm, but it was not signicant ( 58 versus $22 \%, \mathrm{p}=0.09$ ). For letrozole, these results suggest that anastrozole could be prefered to tamoxifen for erbB2-positive tumor patients.

Similarly, a second neoadjuvant comparison of anastrozole and tamoxifen did not show superior response rates when anastrozole was used. ${ }^{34}$ The PROACT trial included 451 postmenopausal women with an ER-positive primary breast cancer. These patients were randomized to receive preoperative anastrozole $(n=228)$ versus tamoxifen $(n=223)$ for a duration of 12 weeks followed by surgery. The primary endpoint of this trial was response rate by ultrasound, defined as shrinkage $>30 \%$ of largest tumor diameter. Response rates were $39.5 \%$ for anastrozole and $35.4 \%$ for tamoxifen $(\mathrm{p}=0.29)$. As 137 patients in this trial received concomitant neoadjuvant chemotherapy, a subgroup analysis of patients on endocrine therapy alone was performed and showed response rates of $36.2 \%$ versus $26.5 \%$ in favor of anastrozole $(\mathrm{p}=0.07)$. Contrary to expectations, the neoadjuvant IMPACT and PROACT studies did not mirror the results generated by the corresponding ATAC adjuvant trial, where anastrozole did prove superior to tamoxifen. ${ }^{35}$ This result can perhaps be explained by the limited duration of therapy in the IMPACT and PROACT studies.

\section{Studies with exemestane (see Table 5)}

Dixon et $\mathrm{al}^{36}$ have evaluated, in a phase II study, the effect of neoadjuvant exemestane in 13 postmenopausal women with histologically or cytologically confirmed breast cancer. Tumors were $3 \mathrm{~cm}$ in diameter, ER+, bidimensionally measurable, operable, and locally advanced. Exemestane $25 \mathrm{mg}$ daily was given for up to 3 months after which definitive surgery was performed. Median reductions in clinical, mammographic and ultrasound tumor volume were $85.5 \%, 84 \%$ and $82.5 \%$, respectively. Before treatment with exemestane, 10/12 patients (1 patient died at the end of the treatment) would have required mastectomy; after treatment, 2 had mastectomy and 10 had breast conservation surgery with clear margins. The most common adverse events were fatigue, insomnia and paresthesia, each reported in 3 patients.

In order to increase the pathological response rate, the exemestane-chemotherapy association as neoadjuvant treatment has been evaluated in 2 phase I studies. ${ }^{37,38}$ Recent preclinical studies provided first evidence that exemestane confers high cytotoxic potential to subtoxic doses of epirubicin when given concomitantly, suggesting that treatment with exemestane and epirubicin might be a safe and effective treatment for breast cancer. Thus, Wolf et al have treated 14 locally advanced breast cancer patients with exemestane $25 \mathrm{mg}$ daily associated with 3 increasing doses of epirubicin (25, 30 and $35 \mathrm{mg} / \mathrm{m}^{2} /$ week) during 8 to 12 weeks. Ten out of 14 patients were evaluable. Wolf et al reported 2 CR, 4 PR, 3 stable diseases and 1 progression. Nine out of 10 patients underwent surgery. A conservative surgery 


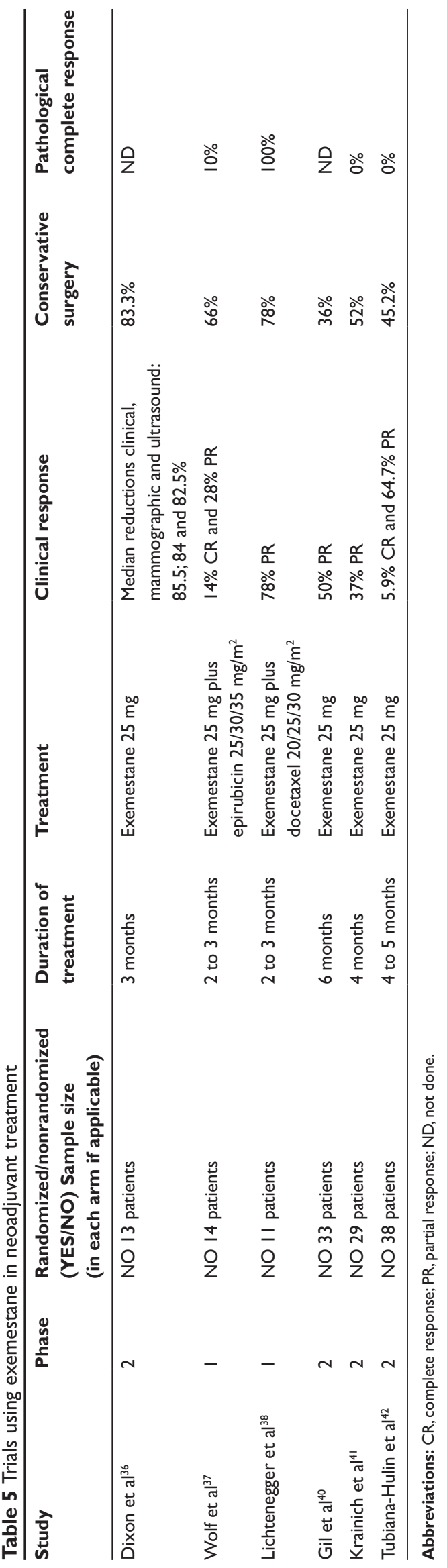

has been conducted on 6 patients $(66 \%)$. Of 10 patients, one presented a complete pathological response and the other presented a partial pathological response. As this combination seemed to be effective, a phase II study has been proposed.

In the same way, Lichtenegger et a ${ }^{138}$ have conducted a phase I study on 11 locally advanced breast cancer patients with exemestane $25 \mathrm{mg}$ daily associated with 3 increasing doses of docetaxel $\left(20,25\right.$ and $30 \mathrm{mg} / \mathrm{m}^{2} /$ week $)$ over 8 to 12 weeks. Nine out of 11 patients were evaluable. They reported $78 \%$ of partial clinical responses and $22 \%$ of stable disease. Pathological response rates were high with $78 \%$ of grade 1 and $22 \%$ of grade 2 , according to the Chevallier et al classification. ${ }^{39}$ As this combination seemed to be effective too, a phase II study has been begun.

Gil et $\mathrm{a}^{40}$ have conducted a phase II study in 33 postmenopausal breast cancer patients. The aim of the study was to evaluate the efficacy of exemestane as neoadjuvant therapy as a primary endpoint. At baseline all patients were considered ineligible for breast-conserving surgery. They were treated with exemestane $25 \mathrm{mg}$ daily for 6 months. The radiological response was measured using RECIST criteria. On the 28 evaluable patients, $50 \%$ were in PR, $45 \%$ presented a stable disease and $5 \%$ were in progression. In all, 15 patients have been operated on: 5 underwent a mastectomy and 10 had a conservative surgery.

In the same way, Krainich et $\mathrm{al}^{41}$ reported a phase II study with exemestane. Twenty-nine patients have been treated with $25 \mathrm{mg}$ daily exemestane for 16 weeks. Twenty-seven patients were evaluable: partial clinical response was registred in $37 \%$ of patients and $63 \%$ of patients presented stable disease. No complete pathological response was registred.

In another phase II study reported by Tubiana-Hulin et $\mathrm{al}^{42} 38$ postmenopausal women with ER-positive operable breast cancer have received 4 to 5 months of neoadjuvant exemestane $(25 \mathrm{mg} /$ day $)$ before surgery. Tumor response was evaluated using RECIST criteria on the first 31 patients: there were $5.9 \%$ of clinically CR, $64.7 \%$ of PR and $23.5 \%$ of stable disease. Breast conserving surgery was achieved in $45.2 \%$.

\section{Discussion and prospects}

According to the literature, neoadjuvant endocrine therapy seems to be effective. The clinical data (overall response rates, conservative surgery) reported when newer generation aromatase inhibitors are used appear to be better than with tamoxifen. Moreover, endocrine therapy is well tolerated. After a review of the literature, several facts merit discussion. 


\section{Hormone receptors}

The data indicate that selection for neoadjuvant endocrine therapy should be based primarily on ER status. Indeed, patients who are treated with neoadjuvant endocrine therapy, with an ER Allred score of 6, and over are most likely to respond and gain a clinical benefit. ${ }^{11}$ Furthermore, aromatase inhibitors produce responses in tumors with lower levels of ER whereas tamoxifen does not..$^{25}$ Thus, it would be interesting to assess the predictive value of ER Allred score as well for tamoxifen and for aromatase inhibitors. These analyses are not are not available will have to be conducted in specific trials. Conversely, according to the study of Buzdar et $\mathrm{al}^{43}$ patients with an ER-positive tumor had a four times higher rate of pathological response to neoadjuvant chemotherapy than patients who with an ER-positive tumor.

\section{Biological data}

Immunohistochemical staining for Ki67 and PgR provided objective measures of major biological effects of therapy and differences between the aromatase inhibitors and tamoxifen. In contrast to tamoxifen, letrozole and anastrozole treatment was associated with a reduction in Ki67 and PgR staining. Although this effect was irrespective of the clinical/ pathological response, ${ }^{23}$ further studies should be conducted with more patients and a to establish a correlation between these biological modifications and response. Moreover, it seems that aromatase inhibitors are effective in both erbB2positive and -negative cancers while tamoxifen is less effective in erbB2-positive tumors. ${ }^{25,33}$ Indeed, in vitro studies have shown evidence for cross-talk and interaction between the ER and Her2 pathways. Previous studies have demonstrated that estrogen downregulates Her2 expression, tamoxifen may upregulate the transcription of Her2, and transfection with the Her 2 coding region in MCF-7 breast cancer cells which are ER-positive and responsive to tamoxifen may lead these cells to become unresponsive to tamoxifen. ${ }^{44-46}$ According to the litterature, there are different causes of resistance: loss or decline of ER expression; ER mutations leading to desensitization to hormonal agents; change in cofactor levels in resistant cells; and Her2 overexpression. From these results, it appears that Her2 plays an important role in resistance to hormonal therapy. Consequently, it would be interesting to evaluate different inhibitors of Her2 and related epidermal growth factor family receptors in association with hormonal therapies.

Other biomarkers could be discovered, particularly for gene expression. Thus and on the basis of preliminary studies, a phase II neoadjuvant letrozole study has been funded by the National Cancer Institute to examine gene expression profiles at baseline, 1 month and at surgery in patients receiving 4 months of endocrine treatment. ${ }^{47}$ The preliminary data show that about 20 genes exhibit a marked decrease in mRNA expression with neoadjuvant letrozole from samples taken at baseline and 1 month from the tumor. These decreases constitute early indices of the effectiveness of endocrine therapy, and the absence of these changes early on in therapy can easily be imagined to be indicative of primary resistance to therapy.

\section{Duration of neoadjuvant therapy}

Standard practice with neoadjuvant chemotherapy is to administer between 3 and 6 cycles prior to surgery, a time period felt sufficient to delineate responders from nonresponders. The optimal duration of neoadjuvant therapy has not been investigated in detail. Nevertheless, Paepke et $\mathrm{al}^{27}$ have reported that patients treated for 8 months with letrozole presented a statistically significant increase in tumor size reduction $(\mathrm{p}=0.0393)$ compared to those who was treated for only 4 months. Furthermore, in another study, ${ }^{34}$ the authors concluded that patients whose tumors are responding to letrozole at 3 months can expect further reduction in tumor volume with continued treatment $(9.5 \%$ $\mathrm{CR}$ at 3 months versus $36 \%$ at 12 months). Then the optimal duration of treatment may be longer than with chemotherapy, the mechanism of action appearing to be linked to a slower process. However, clinical surveillance appears mandatory, as there is always a risk of regression during treatment and before surgery.

For almost all the studies we have mentioned, the follow-up was too short to determine if aromatase inhibitor treatment is associated with any survival or disease-free survival benefit. Such results could be published subsequently. Nevertheless, the population of patients who are treated with neoadjuvant endocrine therapy tends to be elderly and these patients can have significant comorbidity. For many of these patients, despite locally advanced disease, they will die from causes other than breast cancer. For this reason, it could be difficult to assess long term survival of these patients.

\section{Comparison of neoadjuvant endocrine therapy with chemotherapy}

There are no large randomized studies comparing neoadjuvant endocrine therapy with chemotherapy, and little work has been done in this area since the patient populations who are most commonly treated with neoadjuvant chemotherapy tend to be premenopausal women with ER-negative 
tumors. In contrast those treated with endocrine therapy tend to be elderly postmenopausal women with ER-positive tumors. Hence, indications of both therapies are certainly different. Nevertheless, two studies comparing neoadjuvant chemotherapy to neoadjuvant hormonotherapy have been reported by Semiglazov et al ${ }^{48,49}$ Postmenopausal women with ER-positive and/or PgR-positive breast cancer were assigned neoadjuvant treatment with either chemotherapy (doxorubicin and paclitaxel, 4 cycles) or endocrine therapy with aromatase inhibitors (anastrozole or exemestane, 3 months). The overall response rates (clinical and mammography) were statistically similar in the chemotherapy and endocrine groups. Neoadjuvant endocrine therapy with exemestane or anastrozole seems effective and safe in postmenopausal women with ER-positive breast cancer. Such treatment could be a reasonable alternative to chemotherapy for elderly women with ER-positive and/or PgR-positive breast cancer for whom the low toxicity of the regimen is considered an advantage. Further studies would be necessary to confirm these results.

\section{Exploration of neoadjuvant endocrine therapy with chemotherapy}

In the same way, neoadjuvant endocrine therapy has been explored in combination with chemotherapy.

The Genari study group ${ }^{37}$ assessed the impact of a hormone and chemotherapy combination: exemestane associated to epirubicin, or docetaxel, or paclitaxel. Indeed, recent preclinical studies provided first evidence that hormonotherapy has high cytotoxic potential to subtoxic doses of chemotherapy when given concomitantly. Such a combination could potentially increase the $\mathrm{CR}$ rate with the benefit of excellent tolerability.

\section{Exploration of celecoxib and hormone therapy}

The combination of celecoxib (a cyclooxygenase-2 inhibitor) and hormonotherapy needs to be explored. Cyclooxygenases are prostaglandin synthetase enzymes, which play a key role in mammary carcinogenesis. Prostaglandin E2 could contribute to an increase in estrogen synthesis in the tumor. A study was performed to investigate the efficacy of combining anti-aromatase therapy (exemestane and letrozole) and a cyclooxygenase-2 inhibitor (celecoxib) neoadjuvantly in hormone-sensitive postmenopausal breast cancer patients. ${ }^{50,51}$ Postmenopausal women were randomized into three groups. Group A patients received exemestane and celecoxib, group B patients were given exemestane alone and group $\mathrm{C}$ patients received letrozole alone. The maximum duration of treatment was 3 months. The clinical response rates were $61.5 \%, 60 \%$ and $54.5 \%$ respectively for groups $\mathrm{A}, \mathrm{B}$ and $\mathrm{C}$. Although the differences between the three groups were not significant, it would be interesting to conduct further studies to confirm these results. However, the general adversities of cyclooxygenase- 2 inhibitors have to be taken into account.

\section{Interest in neoadjuvant therapy}

Although neoadjuvant chemotherapy has been widely employed to downstage locally advanced-stage breast cancer for quite a while, the use of primary systemic endocrine therapy is still considered something of a novelty. It is not, in fact, a standard treatment but could be useful for elderly women with operable, hormonosensitive, well differentiated and evoluating slowly (SBR I) tumor or for patients with lobular MSBR 1 carcinoma (low chemosensitivity). As for neoadjuvant chemotherapy, neoadjuvant endocrine therapy enables the clinician to test the responsiveness of the malignant cells and provides an early opportunity to change the treatment if the tumor appears resistant. In contrast, toxicity is much lower with neoadjuvant endocrine therapy and this approach is extremely well tolerated, with very few patients having to discontinue the treatment because of side effects.

From a surgical perspective, the ability to perform less extensive surgery is an advantage especially considering the comorbidity and overall general health of the group of patients who tend to be treated with neoadjuvant endocrine therapy. Based on the poor local endocrine control observed in some trials, primary treatment alone without subsequent surgery at all cannot be recommended. ${ }^{14}$ The currently available data suggest that breast-conserving surgery followed by radiotherapy produces adequate local disease control in patients downstaged by neoadjuvant endocrine therapy. ${ }^{52,53}$ The NSABP-B18 study has shown that patients achieving a pCR after neoadjuvant chemotherapy have a significantly better overall survival than the others, suggesting that the achievement of a pCR after neoadjuvant chemotherapy may serve as a surrogate marker for long-term survival. ${ }^{54}$ In contrast to primary chemotherapy, $\mathrm{pCRs}$ for neoadjuvant endocrine therapy is generally considered as an exceptional event. Thus, further investigation would be necessary to determine which parameters may serve as surrogate markers for long-term survival in neoadjuvant endocrine therapy.

After the patient's breast surgery, each case should be discussed in a collegial way for subsequent therapy. Chemotherapy could be proposed especially in case of large 
tumoral residue and node involvement. Radiotherapy should be proposed at least in case of conservative surgery. The continuation of neoadjuvant hormone therapy as adjuvant treatment should be proposed to all patients presenting at least with a stable disease in the neoadjuvant phase.

Results of ongoing trials using neoadjuvant endocrine therapy are awaited with interest.

\section{Disclosures}

The authors disclose no conflicts of interest.

\section{References}

1. Beatson GT. On the treatment of inoperable cases of carcinoma of the mamma: suggestions for a new method of treatment, with illustrative cases. Lancet. 1896;2:104-107.

2. Domont J, Namer M, Khayat D, Spano JP. Neoadjuvant endocrine therapy for breast cancer: an overview. Bull Cancer. 2004;91(1):55-62.

3. King MC, Wieland S, Hale K, Lee M, Walsh T, et al. National surgical adjuvant breast and bowel project. Tamoxifen and breast cancer incidence among women with inherited mutations in BRCA1 and BRCA2: National Surgical Adjuvant Breast and Bowel Project (NSABP-P1). Breast Cancer Prevention Trial. JAMA. 2001;286(18):2251-2256.

4. Powles TJ, Hickish T, Kanis JA, Tidy A, Ashley S. Effect of tamoxifen on bone mineral density measured by dualenergy $\mathrm{x}$-ray absorptiometry in healthy premenopausal and postmenopausal women. J Clin Oncol. 1996;14:78-84.

5. Tamoxifen and endometrial cancer. ACOG committee opinion. No 232 Washington DC: American College of Obstetricians and Gynecologists, 2001. Int J Gynaecol Obstet. 2001;73:77-79.

6. Goss PE, Strasser K. Tamoxifen resistant and refractory breast cancer: the value of aromatase inhibitors. Drugs. 2002;62:957-966.

7. Freedman OC, Verma S, Clemons MJ. Using aromatase inhibitors in the neoadjuvant setting: evolution or revolution? Cancer Treat Rev. 2005;31:1-17.

8. Wells SA, Santen RT, Lipton A, et al. Medical adrenalectomy with aminoglutethemide: clinical studies in postmenopausal patients with metastatic breast carcinoma. Ann Surg. 1978;187:475-484.

9. Stein RC, Dowsett M, Headley A. Treatment of advanced breast cancer in postmenopausal women with 4-hydroxy-androstenedione. Cancer Chemother Pharmacol. 1990;26:75-78.

10. Steele RE, Mellor LB, Swyer WK, Wasvary JM, Browne LJ. In vitro and in vivo studies demonstrating potent and selective oestrogen inhibition with the non-steroidal aromatase inhibitor CGS 16949A Steroids. 1987;50:147-161.

11. Dixon JM, Jackson J, Renshaw L, Miller WR. Neoadjuvant tamoxifen and aromatase inhibitors : comparisons and clinical outcomes. J Steroid Biochem Mol Biol. 2003;86:295-299.

12. Preece PE, Wood RA, Mackie CR, Cuschieri A. Tamoxifen as initial sole treatment of localised breast cancer in elderly women: a pilot study. BMJ. 1982;284:869-870.

13. Howell A, Robertson JFR. The primary use of endocrine therapy. In: Dowsett M, Howell A, Editors. Primary medical therapy for breast cancer: Clinical and biological aspects. ESO Scientific Updates, Amsterdam: Elsevier; 1999. p. 23-37.

14. Tan SM, Cheung KL, Willsher PC, Blamey RW, Chan SB, Robertson JFR. Locally advanced primary breast cancer: mediumterm results of a randomised trial of multimodal therapy versus initial hormone therapy. Eur J Cancer. 2001;37:2331-2338.

15. Mustacchi G, Ceccherini R, Milani S, et al. Tamoxifen alone versus adjuvant tamoxifen for operable breast cancer of the elderly: long-term results of the phase III randomized controlled multicenter GRETA trial. Ann Oncol. 2003;14:414-420.
16. Gazet JC, Ford HT, Coombes RC, et al. Prospective randomised trial of tamoxifen vs. Surgery in elderly patients with breast cancer. Eur $J$ Surg Oncol. 1994;20:207-214.

17. Van Dalsen AD, De Vries J. Treatment of breast cancer in elderly patients. J Surg Oncol. 1995;60:80-82.

18. Bates T, Riley DL, Houghton J, Fallowfield L, Baum M. Breast cancer in elderly women: a Cancer Research Campaign trial comparing treatment with tamoxifen and optimal surgery with tamoxifen alone. Br J Surg. 1991;78:591-594.

19. Early Breast Cancer Trialists' Collaborative Group. Tamoxifen for early breast cancer: an overview of the randomised trials. Lancet. 1998;351:1451-1467.

20. Osborne CK. Steroid hormone receptors in breast cancer management. Breast Cancer Res Treat. 1998;51:227-238.

21. Mauriac L, Debled M, Durand M, Floquet A, Boulanger V, Dagada C. Neoadjuvant tamoxifen for hormone-sensitive non metastatic breast carcinomas in early postmenopausal women. Ann Oncol. 2002;13: 293-298.

22. Dixon JM, Love CDB, Bellamy COC, et al. Letrozole as primary medical therapy for locally advanced and large operable breast cancer. Breast Cancer Res Treat. 2001;66:191-199.

23. Miller WR, Dixon JM, Cameron DA, Anderson TJ. Biological and clinical effects of aromatase inhibitors in neoadjuvant therapy. J Steroid Biochem Mol Biol. 2001;79:103-107.

24. Eiermann W, Paepke S, Appfelstaedt L, et al. Preoperative treatment of postmenopausal breast cancer patients with letrozole: A randomized double-blind multicenter study. Ann Oncol. 2001;12:1505-1506.

25. Ellis MJ, Coop A, Singh B, et al. Letrozole is more effective neoadjuvant endocrine therapy than tamoxifen for ErbB1 and/or ErbB2positive, estrogen receptor- positive primary breast cancer: Evidence from a phase III randomized trial. J Clin Oncol. 2001;19:3808-3816.

26. Wagnerova M, Andrasina I, Sokol L, et al. Our first experience with preoperative treatment of postmenopausal locally advanced breast cancer in postmenopausal patients. J Clin Oncol. Proc Am Soc Clin Oncol. 2002;21:Abstract 156.

27. Paepke S, Tulusan A, Kiesel L, et al. A multi-center study of pre-operative treatment with letrozole for optimal duration of treatment in postmenopausal women with ER and/or PgR positive breast cancer. J Clin Oncol. Proc Am Soc Clin Oncol. 2003. Abstract 321.

28. Renshaw L, Murray J, Young O, et al. Is there an optimal duration of neoadjuvant letrozole therapy? Proceedings 27th Annual San Antonio Breast Cancer Symposium; 2004. Abstract 405.

29. Harvey JM, Clark GM, Osborne CK, Allred DC. Estrogen receptor status by immunohistochemistry is superior to the ligand-binding assay for predicting response to adjuvant endocrine therapy in breast cancer. J Clin Oncol. 1999;17(5):1474-1481.

30. Dixon JM, Renshaw L, Bellamy C, Stuart M, Hoctin-Boes G, Miller WR. The effects of neoadjuvant anastrozole (Arimidex) on tumor volume in postmenopausal women with breast cancer: A randomized, doubleblind, single-center study. Clin Cancer Res. 2000;6:2229-2235.

31. Dixon JM, Jackson J, Hills M, et al. Anastrozole demonstrates clinical and biological effectiveness in erbB2 ER positive breast cancers. Proceedings 25th Annual San Antoninio Breast Cancer Symposium; 2002. Abstract 263.

32. Milla-Santos A, Milla I, Rallo L, Puig-Gali M, Solono V. Anastrozole (A) as neoadjuvant (NEO) therapy for hormone-dependent locally advanced breast cancer (LABC) in postmenopausal (PM) patients (pts). $J$ Clin Oncol. Proc Am Soc Clin Oncol. 2002;21:Abstract 156.

33. Smith I, Dowsett M. Comparison of anastrozole vs tamoxifen alone and in combination as neoadjuvant treatment of oestrogen receptorpositive $(\mathrm{ER}+)$ operable breast cancer in postmenopausal women: the impact trial. Proceedings 26th Annual San Antoninio Breast Cancer Symposium; 2003. Abstract 1.

34. Cataliotti L, Buzdar A, Noguchi S, et al. Efficacy of pre-operative arimidex (anastrozole) compared with tamoxifen (PROACT) as neoadjuvant therapy in postmenopausal women with hormone receptorpositive breast cancer. Eur J Cancer. 2004;2:46. 
35. Howell A, Cuzick J, Baum M, et al. Results of the ATAC (Arimidex, Tamoxifen, Alone or in Combination) trial after completion of 5 years' adjuvant treatment for breast cancer. Lancet. 2005;365:60-62.

36. Dixon JM, Anderson T, Miller WR. Phase IIb study of neoadjuvant exemestane (EXE) in locally advanced breast cancer. J Clin Oncol. Proc Am Soc Clin Oncol. 2001. Abstract 1908.

37. Wolf C, Hackl W, Kuemper S, Schewe T, Eiermann W. Exemestane combined with epirubicin, Q1wX (8-12), as preoperative chemoendocrine treatment for patients with primary breast cancer: a phase I study. J Clin Oncol. Proc Am Soc Clin Oncol. 2001. Abstract 1819.

38. Lichtenegger W, Hackl W, Huettner C, et al. Exemestane combined with weekly docetaxel as preoperative treatment for breast cancer. J Clin Oncol. Proc Am Soc Clin Oncol. 2001. Abstract 1832.

39. Chevallier B, Roche H, Olivier JP, Chollet P, Hurteloup P. Inflammatory breast cancer. Pilot study of intensiveinduction chemotherapy (FEC-HD) results in a high histologic response rate. Am J Clin Oncol. 1993; 16:223-228.

40. Gil M, Barnadas A, Cirera L, et al. Exemestane as neoadjuvant treatment in patients $>65$ years with $\mathrm{T}>3 \mathrm{~cm}$; preliminary results of a multicenter spanish phase II trial. 25th Annual San Antonio Breast Cancer Symposium; 2002. Abstract 272.

41. Krainich U, Astner A, Jonat W, Wallwiener D. Phase II study to define safety and efficacy of exemestane as preoperative therapy for postmenopausal patients with primary breast cancer - final results of the German neoadjuvant aromasin initiative (GENARI). Proceedings 26th Annual San Antonio Breast Cancer Symposium; 2003. Abstract 239.

42. Tubiana-Hulin M, Spyratos F, Becette V, et al. Phase II study of neo-adjuvant exemestane in postmenopausal patients with operable breast cancer. Proceedings 26th Annual San Antonio Breast Cancer Symposium; 2003. Abstract 443.

43. Buzdar AU, Valero V, Therialut RL, et al. Pathological complete response to chemotherapy is related to hormone receptor status. Proceedings 26th Annual San Antonio Breast Cancer Symposium; 2003. Abstract 302.

44. Read LD, Keith D, Slamon J, et al. Hormonal modulation of Her2/neu proto-oncogene messenger ribonucleic acid and p185 protein expression in human breast cancer cell lines. Cancer Res. 1990; 50:3947-3954.
45. Antoniotti S, Maggiora P, Datu V, et al. Tamoxifen upregulates c-erbB-2 transcription in oestrogen-responsive breast cancer cells in vitro. Eur J Cancer. 1992;28:318-321.

46. Benz CC, Scott GK, Sarup JC, et al. Estrogen-dependent, tamoxifenresistant tumorigenic growth of MCF-7 cells, transferred with Her2/neu. Breast Cancer Res Treat. 1992;24:85-95.

47. Ellis MJ, Rosen E, Dressman H, Marks J. Neoadjuvant comparisons of aromatase inhibitors and tamoxifen: pretreatment determinants of response and on-treatment effect. J Steroid Biochem Mol Biol. 2003;86:301-307.

48. Semiglazov VF, Ivanov VG, Semiglazov VV, et al. Neoadjuvant endocrine therapy vs chemotherapy for postmenopausal ER-positive breast cancer patients. Proceedings 27th Annual San Antonio Breast Cancer Symposium; 2004. Abstract 2090.

49. Semiglazov VF, Semiglazov V, Ivanov V, et al. The relative efficacy of neoadjuvant endocrine therapy vs chemotherapy in postmenopausal women with ER-positive breast cancer. J Clin Oncol. Proc Annu Meet Am Soc Clin Oncol. 2004;23. Abstract 519.

50. Chow L, Wong J, Toi M. Celecoxib anti-aromatase neoadjuvant (CAAN) trial for locally advanced breast cancer: preliminary report. J Steroid Biochem Mol Biol. 2003;86:443-447.

51. Chow LWC, Toi M, Takebayashi Y, et al. Celecoxib anti-aromatase neoadjuvant (CAAN) trial for locally advanced breast cancer: second report. Proceedings 26th Annual San Antonio Breast Cancer Symposium; 2003. Abstract 428.

52. Dixon JM. Neoadjuvant therapy: surgical perspectives. In: Miller WR, Ingle JN, eds Endocrine therapy in breast cancer. New York: Marcel Dekker; 2002. p. 197-212.

53. Dixon JM, Anderson TJ, Miller WR. Neoadjuvant endocrine therapy of breast cancer: a surgical perspective. Eur J Cancer. 2002;38: 2214-2221.

54. Fisher B, Bryant J, Wolmark N, et al. Effect of preoperative chemotherapy on the outcome of women with operable breast cancer. J Clin Oncol. 1998;16:2672-2685.
International Journal of General Medicine

\section{Publish your work in this journal}

The International Journal of General Medicine is an international, peer-reviewed open-access journal that focuses on general and internal medicine, pathogenesis, epidemiology, diagnosis, monitoring and treatment protocols. The journal is characterized by the rapid reporting of reviews, original research and clinical studies across all disease areas.

\section{Dovepress}

A key focus is the elucidation of disease processes and management protocols resulting in improved outcomes for the patient. The manuscript management system is completely online and includes a very quick and fair peer-review system. Visit http://www.dovepress.com/ testimonials.php to read real quotes from published authors. 